Research Article

\title{
Intraspecific DNA Barcoding and Variation Analysis for Citri Reticulatae Pericarpium of Citrus reticulata "Chachi"
}

\author{
Mengshi Liu, ${ }^{1}$ Kanghui Wang, ${ }^{1}$ Baizhong Chen, ${ }^{2}$ Yi Cai, ${ }^{1}$ Chuwen Li, ${ }^{1}$ Wanling Yang, \\ Minyan Wei $\mathbb{D}^{1}{ }^{1}$ and Guodong Zheng $\mathbb{D}^{1}$ \\ ${ }^{1}$ Key Laboratory of Molecular Target \& Clinical Pharmacology and the State Key \\ Laboratory of Respiratory Disease \& The Fifth Affiliated Hospital, School of Pharmaceutical Sciences, \\ Guangzhou Medical University, Guangzhou 511436, China \\ ${ }^{2}$ Guangdong Xinbaotang Biological Technology Co., Ltd, Guangdong, Jiangmen 529000, China \\ Correspondence should be addressed to Minyan Wei; weiminyan@163.com and Guodong Zheng; gd200237@126.com
}

Received 26 October 2021; Revised 22 November 2021; Accepted 24 November 2021; Published 9 December 2021

Academic Editor: Francesca Mancianti

Copyright (C) 2021 Mengshi Liu et al. This is an open access article distributed under the Creative Commons Attribution License, which permits unrestricted use, distribution, and reproduction in any medium, provided the original work is properly cited.

\begin{abstract}
Citri Reticulatae Pericarpium, the desiccative mature peel of Citrus reticulata Blanco or its cultivated varieties, is a national geographical indicated product that has the concomitant function of both medicine and foodstuff. The primary source of Citri Reticulatae Pericarpium is Citrus reticulata "Chachi," called "Guang chenpi," while it differs in variety, propagation, grafting rootstock, and tree age, and the hereditary stability of its biological information between intraspecific plants is worthy of our attention. Homologous analysis result of 4 DNA barcodings in the ribosome or the chloroplast showed that the homology of them (ITS2, rbcl, matK, and psbA-trnH) of 22 samples was $100.00 \%, 99.97 \%, 99.99 \%$, and $99.81 \%$, respectively, which indicated that 4 DNA barcodes maintained a high degree of genetic stability in Citrus reticulata "Chachi." Also, ITS2 was considered to identify Citrus reticulata "Chachi" from other varieties because it presented not only low variability within a certain taxon but also a high level of interspecies variability. Simultaneously, variant site detection of Citrus reticulata "Chachi" was analyzed by comparing with the reference Citrus reticulata genome, and 2652697 SNP sites and 533906 InDel sites were detected from whole-genome resequencing data of 22 samples, providing the data resources and theoretical foundation for the future study about the relevant molecular makers of "Guang chenpi."
\end{abstract}

\section{Introduction}

Citri Reticulatae Pericarpium (CRP), a traditional Chinese medicine that has the concomitant function of both medicine and foodstuff, is the desiccated mature peel of Citrus reticulata Blanco or its cultivated varieties. Thereinto, Citrus reticulata "Chachi," the main cultivated variety of Citrus reticulata Blanco, is the primary source of genuine Chinese medicinal materials "Guang Chenpi" [1]. As a national product of geographical indication, "Guang Chenpi" is widely used in clinical applications and by-product processing because of its better quality in CRP [2-4], and most studies have shown that its pharmacological activities include antiasthmatic effects, antineuroinflammatory activity, antioxidant ability, and anticancer activity [5-7].
Up to now, a large number of studies have focused on the chemical compounds in CRP by morphological identification, microscopic identification, TLC, UV, HPLC, GC-MS, and LC-MS [8-10], while these method do not distinguish well among different cultivars or among different varieties of Citrus reticulata "Chachi." As an emerging method of identification of food and natural medicinal materials, molecular marker (DNA barcoding, SNP, and InDel) has considerable untapped potential in the quality control and origin identification of food and medicinal materials. DNA barcoding, an important tool for ecological research, has been widely used in species identification [11-14]. A number of studies have shown that plant DNA core barcodes are used internationally in the fields of species discovery, taxonomy, flora, and ecology [15-17]. Nevertheless, molecular 
marker about different cultivars of CRP or different varieties of Citrus reticulata "Chachi" was less studied. Previous studies have reported that the ITS2 region was selected for discrimination of the four CRP cultivars; however, this study did not take the intraspecific variation of Citrus reticulata "Chachi" into consideration [18]. Between different plants of Citrus reticulata "Chachi," they showed some difference in the tree age and the variety including big-leaf species smallleaf species. Besides, propagations of Citrus reticulata "Chachi" include layerage on its maternal plant or graftage on different rootstocks such as Citrus limonia Osbeck, Citrus reticulata Blanco, and Poncirus trifoliata (L.) Raf.

Herein, 4 DNA barcodings including ITS2, rbcl, matK, and psbA-trnH were chosen for biological evolutionary information analysis about Citrus reticulata "Chachi" of different propagation methods, different tree ages, different varieties, and different rootstocks. Among them, ITS2 is a segment of DNA in the ribosome [19], and $\mathrm{rbcl}$, matK, and psbA-trnH are DNA fragments in the chloroplast.

Except for a study on genetic stability of 4 barcodes, genetic diversity analysis of Citrus reticulata "Chachi" was carried out thought whole-genome resequencing technology with DNBSEQ-T7, compared with the reference published genomic data of Citrus reticulata from the NCBI (GenBank accession number ASM325862v1) [20], further excavating single-nucleotide polymorphism (SNP) sites and insertiondeletion (InDel) sites from whole-genome resequencing data of 22 Citrus reticulata "Chachi" samples.

The objective of this work was to research the hereditary stability of 4 DNA barcodings (ITS2, rbcl, matK, and psbA$\operatorname{trnH}$ ) in different Citrus reticulata "Chachi" plants, which can provide screening indicator of DNA barcoding to distinguish Citrus reticulata "Chachi" and other varieties of CRP. Also, variant type detection based on whole-genome resequencing data provides more potential molecular markers to distinguish Citrus reticulata "Chachi" between intraspecific plants or other cultivars, laying a foundation for the further development of "Guang chenpi."

\section{Materials and Methods}

2.1. Biological Materials. Twenty-two batches of biological materials were collected from the Germplasm Source and Seedling Breeding Center of "Guang chenpi" (Table 1). 22 Citrus reticulata "Chachi" samples were different in variety, plant propagation, rootstock, and tree age.

2.2. DNA Extraction. Genomic DNA was extracted using the plant DNA extraction kit (TSP101-200) of Tsingke. The quality of the extracted genomic DNA was checked by $1 \%$ agarose gel electrophoresis with DL2000 DNA marker, and the concentration of them was carried out through the NanoDrop 1000 (Thermo Fisher Scientific, Waltham Massachusetts, US).

2.3. PCR and Sequencing of DNA Barcodings. Genomic DNA was diluted to $15 \mathrm{ng} \cdot \mu \mathrm{l}^{-1}$ and then was amplified by performing polymerase chain reaction (PCR) using 4 pair of universal primers of DNA barcodings listed in Table 2 [18]. PCR was performed under the following conditions: initial denaturation at $98^{\circ} \mathrm{C}$ for $2 \mathrm{~min}$, followed by 30 cycles with $98^{\circ} \mathrm{C}$ denaturation for $10 \mathrm{~s}$, annealing at the melting temperatures (TM) listed in Table 2 for $10 \mathrm{~s}$, and extension at $72^{\circ} \mathrm{C}$ for $10 \mathrm{~s}$. The final extension step was performed for $5 \mathrm{~min}$ at $72^{\circ} \mathrm{C}$. Next, an aliquot of the amplification product was resolved on $1 \%$ agarose gel electrophoresis documented with a gel documentation system and further analyzed by sequencing.

2.4. DNA Library Construction and Illumina Sequencing. Genomic DNA will be randomly interrupted, the end will be repaired, "A" will be added, and the unique connector of DNBSEQ-T7 sequencer will be added. Then, DNA libraries will be constructed by PCR enrichment. Finally, the DNA library was denatured, cycled, and digested to obtain singlestranded circular DNA. Single-stranded circular DNA was amplified by rolling circle amplification (RCA), further producing DNA nanoball (DNB). Illumina sequencing was performed on DNBSEQ-T7 sequencer after the DNA libraries were qualified.

\subsection{Whole-Genome Resequencing Data Quality and Filtering.} To exclude bias from low-quality reads that arise from the process of base-calling or adapter contamination, the quality of the raw data obtained by whole-genome resequencing was evaluated until the value of $Q_{30}$ was over than $85 \%$. The clean reads were used for subsequent bioinformatics analysis. For further analysis, we downloaded previously published genomic data of Citrus reticulata from the NCBI (GenBank accession number ASM325862v1). We mapped high-quality data per individual to the reference Citrus reticulata genome using Burrows-Wheeler Aligner (BWA) software [21], then the sequencing read depth and genomic coverage of each sample were counted, and the variation was detected.

2.6. SNP and InDel Calling. SNPs and InDels can be called by mapping the unitigs against a reference genome. The main calling procedures are as follows: (1) for the results of BWA comparison, Mark Duplicate tool of Picard software is used to remove the duplication and shield the influence of PCRduplication; (2) the Genome Analysis Toolkit (GATK) software [22] is used to perform InDels realignment, with local realignment of the sites near the alignment result with insertion-miss alignment and correction of alignment errors due to insertion-miss alignment; (3) GATK software was used for base recalibration to calibrate the base masses; (4) variant calling of SNPs and InDels was performed by GATK software; and (5) SNPs and InDels with any of the following features were filtered: two SNPs within $5 \mathrm{bp}$; SNPs within 5 bp near InDel; and two InDels within 10 bp [23].

\section{Results and Discussion}

3.1. Quality and Concentration of the Extracted DNA. In this work, Genomic DNA was extracted from tender leaves of 22 Citrus reticulata "Chachi" samples by using the plant DNA 
TABle 1: Information of Citrus reticulata "Chachi" samples.

\begin{tabular}{|c|c|c|c|c|c|}
\hline No. & Sample source & $\begin{array}{c}\text { Plant } \\
\text { propagation }\end{array}$ & Variety & Rootstock & Tree age (years) \\
\hline A1 & $\begin{array}{l}\text { Tianma Village, Huicheng Town, Xinhui District, } \\
\text { Jiangmen City, Guangdong Province }\end{array}$ & Graftage & Big-leaf & Citrus limonia Osbeck & 11 \\
\hline A2 & $\begin{array}{l}\text { Dadong Village, Huicheng Town, Xinhui District, } \\
\text { Jiangmen City, Guangdong Province }\end{array}$ & Graftage & Big-leaf & Citrus limonia Osbeck & 6 \\
\hline $\mathrm{A} 3$ & $\begin{array}{l}\text { Tianma Village, Huicheng Town, Xinhui District, } \\
\text { Jiangmen City, Guangdong Province }\end{array}$ & Graftage & Big-leaf & Citrus limonia Osbeck & 8 \\
\hline A4 & $\begin{array}{l}\text { Tianma Village, Huicheng Town, Xinhui District, } \\
\text { Jiangmen City, Guangdong Province }\end{array}$ & Graftage & Big-leaf & Citrus limonia Osbeck & 8 \\
\hline A5 & $\begin{array}{l}\text { Dongjia Village, Huicheng Town, Xinhui District, } \\
\text { Jiangmen City, Guangdong Province }\end{array}$ & Layerage & Big-leaf & - & 10 \\
\hline A6 & $\begin{array}{l}\text { Qunsheng Village, Huicheng Town, Xinhui District, } \\
\text { Jiangmen City, Guangdong Province }\end{array}$ & Layerage & Big-leaf & - & 10 \\
\hline A7 & $\begin{array}{l}\text { Qibao Village, Huicheng Town, Xinhui District, } \\
\text { Jiangmen City, Guangdong Province }\end{array}$ & Graftage & Big-leaf & Citrus limonia Osbeck & 11 \\
\hline A8 & $\begin{array}{l}\text { Shenglu Village, Sanjiang Town, Xinhui District, } \\
\text { Jiangmen City, Guangdong Province }\end{array}$ & Graftage & Small-leaf & Citrus limonia Osbeck & 6 \\
\hline A9 & $\begin{array}{l}\text { Guangtian Village, Sanjiang Town, Xinhui District, } \\
\text { Jiangmen City, Guangdong Province }\end{array}$ & Graftage & Wild species & Citrus reticulata Blanco & 5 \\
\hline A10 & $\begin{array}{l}\text { Shenlu Village, Sanjiang Town, Xinhui District, } \\
\text { Jiangmen City, Guangdong Province }\end{array}$ & Graftage & Big-leaf & Citrus limonia Osbeck & 5 \\
\hline A11 & $\begin{array}{c}\text { Shenlu Village, Sanjiang Town, Xinhui District, } \\
\text { Jiangmen City, Guangdong Province }\end{array}$ & Layerage & Big-leaf & - & 5 \\
\hline A12 & $\begin{array}{l}\text { Xinsheng Village, Siqian Town, Xinhui District, } \\
\text { Jiangmen City, Guangdong Province }\end{array}$ & Graftage & Big-leaf & Citrus limonia Osbeck & 25 \\
\hline A13 & $\begin{array}{l}\text { Shanyi Village, Siqian Town, Xinhui District, } \\
\text { Jiangmen City, Guangdong Province }\end{array}$ & Graftage & Small-leaf & Citrus limonia Osbeck & 10 \\
\hline A 14 & $\begin{array}{l}\text { Shanyi Village, Siqian Town, Xinhui District, } \\
\text { Jiangmen City, Guangdong Province }\end{array}$ & Graftage & Small-leaf & Citrus limonia Osbeck & 10 \\
\hline A15 & $\begin{array}{l}\text { Shanyi Village, Siqian Town, Xinhui District, } \\
\text { Jiangmen City, Guangdong Province }\end{array}$ & Graftage & Big-leaf & Poncirus trifoliata (L.) Raf. & 8 \\
\hline A 16 & $\begin{array}{l}\text { Yaqian Village, Shuangshui Town, Xinhui District, } \\
\text { Jiangmen City, Guangdong Province }\end{array}$ & Graftage & Big-leaf & Citrus limonia Osbeck & 8 \\
\hline A17 & $\begin{array}{l}\text { Shalu Village, Shuangshui Town, Xinhui District, } \\
\text { Jiangmen City, Guangdong Province }\end{array}$ & Graftage & Big-leaf & Citrus limonia Osbeck & 5 \\
\hline A18 & Guangdong Province seedling breeding farm & Graftage & Big-leaf & Poncirus trifoliata (L.) Raf. & 10 \\
\hline A19 & Guangdong Province seedling breeding farm & Graftage & Big-leaf & Citrus limonia Osbeck & 6 \\
\hline A20 & $\begin{array}{l}\text { Wenlong Village, Daze Town, Xinhui District, } \\
\text { Jiangmen City, Guangdong Province }\end{array}$ & Graftage & Big-leaf & Citrus limonia Osbeck & 10 \\
\hline $\mathrm{A} 21$ & $\begin{array}{c}\text { Yaxi Village, Yamen Town, Xinhui District, Jiangmen } \\
\text { City, Guangdong Province }\end{array}$ & Graftage & Big-leaf & Citrus limonia Osbeck & 13 \\
\hline $\mathrm{A} 22$ & $\begin{array}{l}\text { Changsha Village, Gujing Town, Xinhui District, } \\
\text { Jiangmen City, Guangdong Province }\end{array}$ & Graftage & Big-leaf & Citrus limonia Osbeck & 10 \\
\hline
\end{tabular}

TABle 2: Primer sequence and PCR system of DNA barcodings.

\begin{tabular}{lccc}
\hline Barcoding & Direction & Primer sequence $\left(5^{\prime} \longrightarrow 3^{\prime}\right)$ & PCR system \\
\hline \multirow{2}{*}{ ITS2 } & $\mathrm{F}$ & ATGCGATACTTGGTGTGAAT & \\
& $\mathrm{R}$ & GACGCTTCTCCAGACTACAAT $\left({ }^{\circ} \mathrm{C}\right)$ \\
$\operatorname{rbcl}$ & $\mathrm{F}$ & ATGTCACCACAAACAGAAAC & 58 \\
& $\mathrm{R}$ & TCGCATGTACCTGCAGTAGC & $98^{\circ} \mathrm{C} 2 \mathrm{~min} ;$ \\
matK & $\mathrm{F}$ & AGAGGTATTTGCTGCTGTGGTG & $98^{\circ} \mathrm{C} 10 \mathrm{~s}, \mathrm{TM}{ }^{\circ} \mathrm{C} 10 \mathrm{~s}, 72^{\circ} \mathrm{C} 10 \mathrm{~s} ;$ \\
& $\mathrm{R}$ & GGAAAGAGTAAAGCAAGAACGTGT & $72^{\circ} \mathrm{C} 5 \mathrm{~min}$ \\
psbA-trnH & $\mathrm{F}$ & AGGTATCTGGTTCACTGCTTTAGGT & 58 \\
& $\mathrm{R}$ & GCCTTGATCCACTTGGCTACAT & 59 \\
\hline
\end{tabular}

TM, The melting temperature of DNA. 
extraction kit, and OD value (A260/280) and DNA concentration are shown in Table 3. The results showed that the concentration of DNA could be used in subsequent experiments.

3.2. Sequence Features and Homologous Analysis of DNA Barcodings. According to the agarose gel electrophoresis result of PCR amplification products (Figure 1), ITS2, rbcl, matK, and psbA-trnH produced amplification bands of approximately $750 \mathrm{bp}, 750 \mathrm{bp}, 1000 \mathrm{bp}$, and $500 \mathrm{bp}$, respectively. The electrophoresis bands of each sample were uniform, bright, and nonspecific heterozygous, indicating that the success rate of sequence amplification was $100 \%$, which could be further analyzed by sequencing.

Four barcodes (ITS2, rbcl, matK, and psbA-trnH) were analyzed by DNAMAN software for the length and base composition of each sequence fragment and further identified by BLAST in Genebank. The success rates of PCR amplification and sequencing of 3 DNA barcodings (ITS2, rbcl, and matK) of 22 samples were $100 \%$. However, due to the large number of fragments missing in the sequencing of two samples (A16 and A21) of the psbA-trnH barcode, 20 psbA-trnH sequences were actually obtained in the experiment. PCR success rate, barcoding length, GC content, variable site, and BLAST rate of 4 DNA barcodings are listed in Table 4. The aligned partial sequences had lengths of $232 \mathrm{bp}, 680 \sim 682 \mathrm{bp}, 1059 \mathrm{bp}$, and 537 538 bp for ITS2, rbcl, matK, and psbA-trnH, respectively. Among them, the ITS2 barcode had the advantages of shorter sequence length and higher GC content (71.60\%), followed by the psbA-trnH barcode having shorter sequence length.

Moreover, homologous analysis of 4 DNA barcodings about 22 batches of Citrus reticulata "Chachi" samples was carried out by DNAMAN software. The homologous analysis result showed that the homology of ITS2 of 22 samples was $100.00 \%$, which indicated that ITS2 maintained a high degree of genetic stability in Citrus reticulata "Chachi" of different propagation methods, different tree ages, different varieties, and different rootstocks. Also, the homology of rbcl, matK, and psbA-trnH of 22 samples was 99.97\%, $99.99 \%$, and $99.81 \%$, respectively.

In addition, among the 22 batches of CRP samples, 2 SNP sites were identified in the matK barcode and 1 Indel site was identified in the psbA-trnH barcode. The results showed that there were still some variations within the species of Citrus reticulata "Chachi," making us realize molecular breeding of Citrus reticulata "Chachi" and the distinction of Citrus reticulata "Chachi" and related species need more valuable molecular markers. Therefore, wholegenome resequencing was also performed on 22 Citrus reticulata "Chachi" samples of different propagation methods, different tree ages, different varieties, and different rootstocks, which provided more scientific basis for molecular breeding.

Previous studies have reported that the ITS2 region was selected for discrimination of the four CRP cultivars including Citrus reticulata "Chachi," Citrus reticulata "Dahongpao," Citrus reticulata "Unshiu," and Citrus reticulata "Tangerina," while ITS, trnH-psbA, and rbcL could not distinguish these CRP samples [18]. Different from the existing studies, this work focuses on the hereditary stability of 4 barcodes including ITS2, rbcl, matK, and psbA-trnH in Citrus reticulata "Chachi" with different varieties, propagation methods, grafting rootstocks, and tree ages. Because of DNA degradation in moderately or highly processed products with time, PCR amplification of standard-length (around $650 \mathrm{bp}$ ) barcodings is a huge challenge [24]. Combined with existing research and the result in this work, ITS2 was considered to be a useful DNA barcoding to distinguish Citrus reticulata "Chachi" from other varieties, which presented not only low variability within a certain taxa but also a high level of interspecies variability. Also, this work indicated that matK was not considered because of its long length and variable sites within taxa, while rbcl and psbA-trnH had the potential to distinguish Citrus reticulata "Chachi" from other varieties. Actually, combining DNA barcodes in the ribosome and in the chloroplast makes it more convincing in species identification of plants $[25,26]$.

3.3. Quality Analysis of Whole-Genome Resequencing Data. A total of 22 Citrus reticulata "Chachi" sample genomes were sequenced, which generated $158 \mathrm{~Gb}$ raw data. Base coverage depth distribution curve and coverage distribution curve indicated that the coverage depth of the bases on the genome was evenly distributed. The statistical results of insert fragment distribution with a single peak show that insert fragment distribution fits the normal distribution and the construction of DNA libraries was reliable. The chromosome coverage depth map showed that the genome was evenly covered, indicating good randomness of sequencing.

Summary of clean sequencing data results about 22 Citrus reticulata "Chachi" samples is given in Table 5. The size of reference genome Citrus reticulata is $344.27 \mathrm{Mb}$ (assembly level: scaffold). In this work, the average coverage depth was $16 \mathrm{X}$, and the value of $Q_{30}$ reached $88.94 \%$. The average mapped ratio and genome coverage of all the samples were $98.97 \%$ and $93.55 \%$, respectively. The average GC content of Citrus reticulata "Chachi" was $38.68 \%$ in line with reference genome Citrus reticulata.

3.4. SNP and InDel Calling of Citrus reticulata "Chachi". In this study, the variant site detection of Citrus reticulata "Chachi" for the national geographical indicated product CRP was firstly analyzed by SNP and InDel calling from whole-genome resequencing data (Table 6). Except for the high genetic stability of 4 barcodes (ITS2, rbcl, matK, and psbA-trnH), 22 Citrus reticulata "Chachi" samples showed its genetic diversity between different propagation methods, different tree ages, different varieties, and different rootstocks as well.

A total of 2652697 SNP sites were excavated between 22 Citrus reticulata "Chachi" samples, among which 1741507 SNP sites were transition $\left(\mathrm{T}_{\mathrm{i}}\right), 902182$ SNP sites were transversion $\left(\mathrm{T}_{\mathrm{v}}\right)$, and $9008 \mathrm{SNP}$ sites were transition or transversion. These SNP sites were with a $\mathrm{T}_{\mathrm{i}} / \mathrm{T}_{\mathrm{v}}$ ratio of 1.93, 
TABLE 3: Quality and concentration of the extracted DNA.

\begin{tabular}{lccc}
\hline Sample no. & OD value $(\mathrm{A} 260 / 280)$ & DNA concentration $(\mathrm{ng} / \mu \mathrm{l})$ & Total DNA $(\mu \mathrm{g})$ \\
\hline A1 & 1.792 & 178 & 151 \\
$\mathrm{~A} 2$ & 1.763 & 104 & 4.34 \\
$\mathrm{~A} 3$ & 1.671 & 240 & 3.12 \\
$\mathrm{~A} 4$ & 1.749 & 110 & 7.20 \\
$\mathrm{~A} 5$ & 1.790 & 16 & 3.30 \\
$\mathrm{~A} 6$ & 1.115 & 153 & 0.47 \\
$\mathrm{~A} 7$ & 1.826 & 104 & 4.59 \\
A8 & 1.761 & 134 & 3.12 \\
A9 & 1.707 & 186 & 4.02 \\
A10 & 1.788 & 150 & 5.58 \\
A11 & 1.826 & 11 & 4.50 \\
A12 & 1.882 & 125 & 0.32 \\
A13 & 1.785 & 179 & 3.75 \\
A14 & 1.805 & 133 & 5.37 \\
A15 & 1.816 & 166 & 3.99 \\
A16 & 1.812 & 149 & 4.98 \\
A17 & 1.817 & 81 & 4.47 \\
A18 & 1.656 & 112 & 2.42 \\
A19 & 1.811 & 144 & 3.36 \\
A20 & 1.818 & 236 & 7.32 \\
A21 & 1.740 & 120 & 3.08 \\
A22 & 1.650 & & 3.60 \\
\hline
\end{tabular}

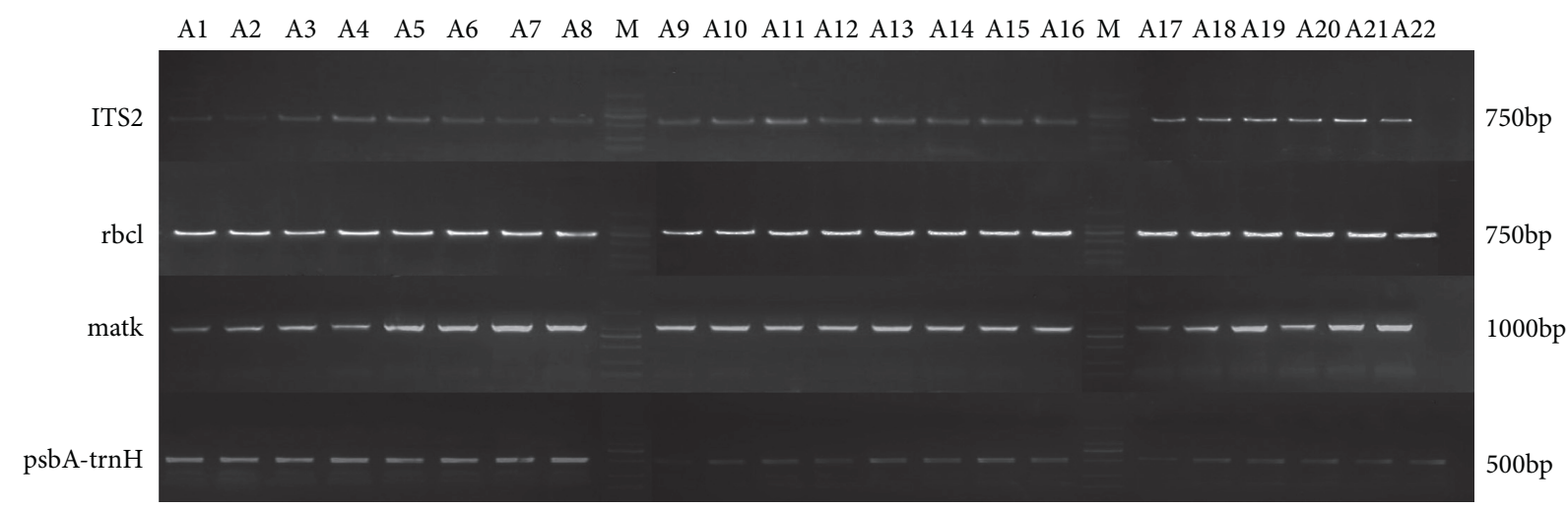

FIGURE 1: Agarose gel electrophoresis of ITS2, rbcl, matK, and psbA-trnH.

TABle 4: Sequence features of 4 DNA barcodings.

\begin{tabular}{lccccc}
\hline Barcoding & PCR success rate (\%) & Barcoding length $(\mathrm{bp})$ & $G+C$ content $(\%)$ & Variable site & BLAST rate $(\%)$ \\
\hline ITS2 & 100 & 232 & 71.60 & 0 & 100.00 \\
rbcl & 100 & $680 \sim 682$ & 44.80 & 0 & 99.79 \\
matK & 100 & 1059 & 36.00 & 2 & 100.00 \\
psbA-trnH & 100 & $537 \sim 538$ & 32.46 & 1 & 99.91 \\
\hline
\end{tabular}

which is in line with general rules of base mutation in natural organisms [27]. In the course of evolution about Citrus reticulata "Chachi," transition happens much more frequently than transversion, which means that evolution of Citrus reticulata "Chachi" tends to accept the substitution between purines and purines or the substitution between pyrimidines and purines, the substitution between purines and pyrimidines causes bad things to happen, and that substitution has mostly been eliminated by evolution.
In addition, InDel sites, as codominant molecular markers, are widely distributed in the genome with high density, which are suitable for genome-wide molecular marker exploration. A total of 533906 InDel sites were detected between 22 Citrus reticulata "Chachi" samples, among which 275380 InDel sites were insertion, 241768 InDel sites were deletion, and $9008 \mathrm{InDel}$ sites were insertion or deletion. Unlike SNP sites, insertions and deletions of InDel sites are equally likely to occur. 
TABLE 5: Summary of sequencing data results about Citrus reticulata "Chachi."

\begin{tabular}{|c|c|c|c|c|c|c|c|c|}
\hline No. & Clean reads & Clean bases & Depth $(\mathrm{X})$ & $Q_{20}(\%)$ & $Q_{30}(\%)$ & $G+C$ content $(\%)$ & Read mapping (\%) & Genome mapping (\%) \\
\hline A1 & 49667300 & 7450095000 & 17 & 96.64 & 89.37 & 39.04 & 99.06 & 94.65 \\
\hline A2 & 45743350 & 6861502500 & 16 & 95.80 & 87.28 & 38.13 & 98.54 & 92.53 \\
\hline A3 & 47316474 & 7097471100 & 17 & 96.33 & 88.37 & 37.91 & 98.98 & 93.78 \\
\hline A4 & 41918262 & 6287739300 & 15 & 96.35 & 88.30 & 38.14 & 98.83 & 93.42 \\
\hline A5 & 44889792 & 6733468800 & 16 & 96.25 & 88.36 & 38.03 & 98.65 & 93.06 \\
\hline A6 & 32910626 & 4936593900 & 11 & 95.86 & 87.12 & 38.51 & 98.86 & 93.17 \\
\hline A7 & 45716786 & 6857517900 & 16 & 96.07 & 88.12 & 39.22 & 99.18 & 94.68 \\
\hline A8 & 80297744 & 112044661600 & 29 & 96.35 & 89.09 & 38.45 & 99.29 & 93.83 \\
\hline A9 & 49858294 & 7478744100 & 15 & 96.71 & 89.57 & 40.23 & 98.81 & 93.39 \\
\hline A10 & 43580158 & 6537023700 & 15 & 96.22 & 88.29 & 39.44 & 99.05 & 94.45 \\
\hline A11 & 46206262 & 6930939300 & 16 & 96.72 & 89.52 & 38.76 & 98.85 & 93.56 \\
\hline A12 & 42274388 & 6341158200 & 15 & 96.74 & 91.56 & 38.15 & 99.02 & 88.15 \\
\hline A13 & 53238294 & 7985744100 & 18 & 96.36 & 88.47 & 38.62 & 99.03 & 93.94 \\
\hline A14 & 42326498 & 6348974700 & 15 & 96.40 & 88.89 & 39.60 & 99.24 & 94.72 \\
\hline A15 & 47956602 & 7193490300 & 17 & 96.52 & 88.85 & 38.43 & 99.04 & 94.23 \\
\hline A16 & 47127502 & 7069125300 & 16 & 96.30 & 88.36 & 38.03 & 98.70 & 92.89 \\
\hline A17 & 45382574 & 6807386100 & 16 & 96.37 & 88.52 & 39.02 & 99.17 & 94.85 \\
\hline A18 & 53972916 & 8095937400 & 20 & 96.33 & 88.78 & 37.67 & 99.15 & 94.28 \\
\hline A19 & 48034834 & 7205225100 & 17 & 95.99 & 87.54 & 38.21 & 99.05 & 93.35 \\
\hline $\mathrm{A} 20$ & 43896212 & 6584431800 & 15 & 96.55 & 88.99 & 38.44 & 98.84 & 93.40 \\
\hline A21 & 45485090 & 6822763500 & 15 & 96.42 & 88.64 & 39.89 & 99.10 & 94.31 \\
\hline A22 & 43174144 & 6476121600 & 14 & 96.74 & 89.42 & 38.98 & 98.96 & 93.62 \\
\hline
\end{tabular}

TABle 6: SNPs and InDels of Citrus reticulata "Chachi."

\begin{tabular}{|c|c|c|c|c|}
\hline Variant type & Category & Number & Category/variant type (\%) & Total \\
\hline \multirow{3}{*}{ SNP } & Transition & 1741507 & 65.65 & \multirow{3}{*}{2652697} \\
\hline & Transversion & 902182 & 34.01 & \\
\hline & Transition/transversion & 9008 & 0.34 & \\
\hline \multirow{3}{*}{ InDel } & Insertion & 275380 & 51.58 & \multirow{3}{*}{533906} \\
\hline & Deletion & 241768 & 45.28 & \\
\hline & Insertion/deletion & 16758 & 3.14 & \\
\hline
\end{tabular}

\section{Conclusions}

Overall, our work indicated that 4 DNA barcodes (ITS2, rbcl, matK, and psbA-trnH) maintained a high degree of genetic stability in Citrus reticulata "Chachi" of different propagation methods, different tree ages, different varieties, and different rootstocks. Because ITS2 presented not only low variability within a certain taxa but also a high level of interspecies variability, it was considered to be an useful DNA barcoding to identify Citrus reticulata "Chachi" from other varieties. Moreover, 2652697 SNP sites and 533906 InDel sites were detected from wholegenome resequencing data of 22 Citrus reticulata "Chachi" samples, fully reflecting the genetic diversity of Citrus reticulata "Chachi" with different varieties or propagation methods. To excavate more useful molecular markers for distinguishing Citrus reticulata "Chachi" between intraspecific plants or other cultivars, DNA barcoding analysis and variant type detection of the Citrus reticulata "Chachi" were studied for the first time in this investigation, which laid a special foundation for the biological information analysis of Citrus reticulata "Chachi" for the national geographical indicated product Citri Reticulatae Pericarpium.

\section{Data Availability}

The data used to support the findings of this study are included within the article.

\section{Conflicts of Interest}

The authors declare no conflicts of interest.

\section{Authors' Contributions}

Mengshi Liu and Kanghui Wang conceptualized the study, performed visualization, conducted investigation, and wrote the original draft of the manuscript. Baizhong Chen, Yi Cai, Chuwen Li, and Wanling Yang formulated the methodology, were responsible for software, and curated data. Minyan Wei and Guodong Zheng formulated the methodology, were responsible for software, curated data, performed validation, supervised the work, and reviewed and edited the manuscript. All authors agreed to be accountable for all aspects of work ensuring integrity and accuracy. Mengshi Liu and Kanghui Wang contributed equally to this work. 


\section{Acknowledgments}

This work was primarily supported by the National Key R\&D Program of China (Nos. 2017YFC1701103 and 2017YFC1701105), Technology Plan of Zhuhai in 2020 (No. ZH22036207200009PWC), Cultivation Plan for High-Level University Academic Backbone of Guangzhou Medical University in 2017 (No. gydf [2017] 210), and High-level University Construction Fund of Guangdong Province (06-410-2107235).

\section{References}

[1] Committee of National Pharmacopoeia, The Pharmacopoeia of the People's Republic of China, China Medical Science and Technology Press, Beijing, China, 2020.

[2] M. Fu, B. Zou, K. An et al., "Anti-asthmatic activity of alkaloid compounds from pericarpium Citri reticulatae (Citrus reticulata "Chachi")," Food \& Function, vol. 10, no. 2, pp. 903-911, 2019.

[3] J. Guo, H. Tao, Y. Cao, C.-T. Ho, S. Jin, and Q. Huang, "Prevention of obesity and type 2 diabetes with aged Citrus peel (chenpi) extract," Journal of Agricultural and Food Chemistry, vol. 64, no. 10, pp. 2053-2061, 2016.

[4] J.-J. Xu, X. Wu, M.-M. Li et al., "Antiviral activity of polymethoxylated flavones from "Guang chenpi", the edible and medicinal pericarps of Citrus Reticulata "Chachi"," Journal of Agricultural and Food Chemistry, vol. 62, no. 10, pp. 2182-2189, 2014.

[5] S.-C. Ho and C.-T. Kuo, "Hesperidin, nobiletin, and tangeretin are collectively responsible for the anti-neuroinflammatory capacity of tangerine peel (Citri reticulatae pericarpium)," Food and Chemical Toxicology, vol. 71, pp. 176-182, 2014.

[6] M. Fu, Y. Xu, Y. Chen et al., "Evaluation of bioactive flavonoids and antioxidant activity in Pericarpium Citri Reticulatae ( Citrus reticulata "Chachi") during storage," Food Chemistry, vol. 230, pp. 649-656, 2017.

[7] G. D. Zheng, P. J. Hu, Y. X. Chao et al., "Nobiletin induces growth inhibition and apoptosis in human nasopharyngeal carcinoma C666-1 cells through regulating PARP -2/SIRT 1/ AMPK signaling pathway," Food Sciences and Nutrition, vol. 7, no. 3, pp. 1104-1112, 2019.

[8] L. Duan, L. Guo, L.-L. Dou et al., "Discrimination of Citrus reticulata Blanco and Citrus reticulata "Chachi" by gas chromatograph-mass spectrometry based metabolomics approach," Food Chemistry, vol. 212, pp. 123-127, 2016.

[9] M. Luo, H. Luo, P. Hu, Y. Yang, B. Wu, and G. Zheng, "Evaluation of chemical components in Citri Reticulatae Pericarpium of different cultivars collected from different regions by GC-MS and HPLC," Food Sciences and Nutrition, vol. 6, no. 2, pp. 400-416, 2017.

[10] G. Zheng, M. Liu, Y. Chao et al., "Identification of lipophilic components in Citri Reticulatae Pericarpium cultivars by supercritical CO 2 fluid extraction with ultra-highperformance liquid chromatography-Q Exactive Orbitrap tandem mass spectrometry," Journal of Separation Science, vol. 43, no. 17, pp. 3421-3440, 2020.

[11] Z. Gao, Y. Liu, X. Wang, X. Wei, and J. Han, "DNA minibarcoding: a derived barcoding method for herbal molecular identification," Frontiers of Plant Science, vol. 10, p. 987, 2019.

[12] Q.-j. Li, X. Wang, J.-r. Wang et al., "Efficient identification of pulsatilla (Ranunculaceae) using DNA barcodes and micro-morphological Characters," Frontiers of Plant Science, vol. 10, p. 1196, 2019.

[13] J. Chen, Y. B. Zhao, Y. J. Wang, and X. G. Li, "Identification of species and materia medica within Saussurea subg. Amphilaena based on DNA barcodes," PeerJ, vol. 7, p. e6357, 2019.

[14] J. Yu, X. Wu, C. Liu, S. Newmaster, S. Ragupathy, and W. J. Kress, "Progress in the use of DNA barcodes in the identification and classification of medicinal plants," Ecotoxicology and Environmental Safety, vol. 208, Article ID 111691, 2021.

[15] P. M. Hollingsworth, D.-Z. Li, M. van der Bank, and A. D. Twyford, "Telling plant species apart with DNA: from barcodes to genomes," Philosophical Transactions of the Royal Society B: Biological Sciences, vol. 371, no. 1702, Article ID 20150338, 2016.

[16] S.-J. Hu, H.-Y. Hu, H. Gao, X. Liu, and S.-L. Chen, "DNA barcoding and rapid identification of the precious herb Herba Anoectochili," Chinese Journal of Natural Medicines, vol. 17, no. 10, pp. 738-745, 2019.

[17] W. J. Kress, C. García-Robledo, M. Uriarte, and D. L. Erickson, "DNA barcodes for ecology, evolution, and conservation," Trends in Ecology \& Evolution, vol. 30, no. 1, pp. 25-35, 2015.

[18] S.-Z. Li, S.-L. Zeng, Y. Wu et al., "Cultivar differentiation of Citri Reticulatae Pericarpium by a combination of hierarchical three-step filtering metabolomics analysis, DNA barcoding and electronic nose," Analytica Chimica Acta, vol. 1056, pp. 62-69, 2019.

[19] J. Liu, L. Shi, J. Han et al., "Identification of species in the angiosperm family Apiaceae using DNA barcodes," Molecular Ecology Resources, vol. 14, no. 6, pp. 1231-1238, 2014.

[20] L. Wang, F. He, Y. Huang et al., "Genome of wild Mandarin and domestication history of Mandarin," Molecular Plant, vol. 11, no. 8, pp. 1024-1037, 2018.

[21] H. Li and R. Durbin, "Fast and accurate short read alignment with Burrows-Wheeler transform," Bioinformatics, vol. 25, no. 14, pp. 1754-1760, 2009.

[22] A. McKenna, M. Hanna, E. Banks et al., "The Genome Analysis Toolkit: a MapReduce framework for analyzing nextgeneration DNA sequencing data," Genome Research, vol. 20, no. 9, pp. 1297-1303, 2010.

[23] J. Reumers, P. De Rijk, H. Zhao et al., "Optimized filtering reduces the error rate in detecting genomic variants by shortread sequencing," Nature Biotechnology, vol. 30, no. 1, pp. 61-68, 2011.

[24] K. Böhme, P. Calo-Mata, J. Barros-Velázquez, and I. Ortea, "Review of recent DNA-based methods for main foodauthentication topics," Journal of Agricultural and Food Chemistry, vol. 67, no. 14, pp. 3854-3864, 2019.

[25] D. Teske, A. Peters, A. Möllers, and M. Fischer, "Genomic profiling: the strengths and limitations of chloroplast genomebased plant variety authentication," Journal of Agricultural and Food Chemistry, vol. 68, no. 49, pp. 14323-14333, 2020.

[26] N. Kane, S. Sveinsson, H. Dempewolf et al., "Ultra-barcoding in cacao (Theobromaspp.; Malvaceae) using whole chloroplast genomes and nuclear ribosomal DNA," American Journal of Botany, vol. 99, no. 2, pp. 320-329, 2012.

[27] S. Duchêne, S. Y. Ho, and E. C. Holmes, "Declining transition/ transversion ratios through time reveal limitations to the accuracy of nucleotide substitution models," BMC Evolutionary Biology, vol. 15, no. 1, p. 36, 2015. 